\title{
A Study on Segmenting Brain Tumor MRI Images
}

\author{
1N. Nazeeya Anjum, \\ ${ }^{1}$ Assistant Professor, Dept of ECE, Sri Sairam Engineering College, \\ Chennai, Tamil Nadu, India. \\ "*orresponding Author: nazeeyaanjum.ece@sairam.edu.in
}

Received: 15.01.2021, Revised: 05.03 .2021 , Accepted: 05.04.2021, Published: 16.04 .2021

DOI:

10.53409/mnaa/jcsit/2101

Abstract: Segmentation of image has traditionally been referred to as the initial stage in image processing. A successful segmentation output will make image processing analysis considerably further easier. There are several image segmentation techniques and methodologies available. Clustering is the most widely used segmentation algorithm for image processing. Segmentation of tumor using magnetic resonance imaging (MRI) data is a critical procedure yet time-consuming process manually carried out by medical specialists. Due to the considerable difference in the tumor tissue appearances across patients, as well as their occasionally similar resemblance to normal tissues, automating this procedure is difficult. MRI is a sort of sophisticated medical imaging that offers precise information on the human soft tissues. To identify and segment the brain tumor using MRI images, several brain tumor segmentation and detection approaches are analyzed. The benefits and drawbacks of these approaches for brain tumor identification and segmentation are analyzed, with an emphasis on illuminating the benefits and limitations of these techniques for brain tumor segmentation and detection. The MRI image usage in segmentation and detection on various processes is also covered. This analysis provides an overview of several segmentation methods for identifying brain tumors from MRI images of the brain, as well as the usage of various Clustering Techniques.

Keywords: Tumor Detection; Magnetic Resonance Imaging (MRI); Tumor Segmentation, Automated System, Pre-processing, Filtering.

\section{INTRODUCTION}

egmentation is a method that divides an image into numerous segments to accurately identify its pixels in a decisionoriented application. It splits an image into many discrete areas, with strong similarity and contrast between the pixels in each zone. As a result, segmentation of image is used to change or simplify the image's representation, or to turn the features of an image into a most useful form, making it easier to study. It is a helpful tool in a variety of fields and applications, including medical image processing, health care, traffic image analysis, pattern identification, and so on.

Image segmentation may be accomplished using a variety of approaches and methodologies, including graph-based, threshold-based, and morphologically based, edges based, clustering-based, neural networkbased, and etc. Among these strategies, clustering is one of the most commonly utilized and successful. These techniques each have their own set of benefits and drawbacks, therefore one must select an algorithm depending on their own needs. There are several clustering procedures, such as Fuzzy C-means clustering, K-means clustering, Subtractive clustering approaches, and so on.

In this study, a review was done on various clustering strategies and recent researches made on them. MRI of brain image computation has dramatically expanded the domain of medicine by offering various approaches for extracting and visualizing data from medical images obtained through different retrieval conditions. The method of extracting data from complex MRI brain images is known as brain tumor segmentation. In today's medical world, diagnostic imaging is a very valuable method. Imaging techniques such as MRI, computed tomography (CT), digital mammography, and others are useful in detecting various diseases. When the number of patients rises, automated detection methodologies enhance information of normal and abnormal examinations for medical testing and play a major role in diagnosis and 
treatments. For evaluating brain MRI, anatomical structures like muscles, bones, tissue types, blood vessels, pathological regions like multiple sclerosis lesions, cancer, and splitting a whole image into sub-regions like grey matter (GM), white matter (WM), and cerebrospinal fluids (CS), segmentation has a wide range of applications in medical imaging. Thus, segmentation of brain tumor is important in MRI. MRI is especially well suited to brain studies due to its great contrast of soft issues, high spatial resolutions, and non-invasive nature. Segmentation of brain tumor divides a part of the image into collectively unique and channeled areas, ensuring that every ROI is locally contiguous and that the pixels inside the areas are homogeneous according to a predefined condition. Texture, concentration, color, surface normal, range, and surface curvature are examples of homogeneity conditions.

Many researchers have prepared important research in the field of brain tumor segmentation in the past, and it remains one of the most critical research topics today. Medical history, imaging scans, biopsy (a tiny brain tissue is removed and examined using microscope) are all essential for the brain tumor analysis. The diagnostic process can begin with standard x-rays and computed tomography (CT). On the other hand, MRI is very valuable since it offers more comprehensive details regarding the tumor types, location, and sizes. As a result, MRI is a better option for the diagnostic workup, surgery and procedure outcome monitoring.

\section{RELATED WORK}

In anomaly prediction, inaccuracies were a major issue in the medical image processing field. These issues have increased as a result of errors generated by the operator, device/instruments, and environment, all of which may be addressed using the innovative segmentation approach presented in this study. To correct the inaccurate predictions of anomalies in different topographical locations in MRI brain subjects, in [1] a new method combined the functions of the Interval Type-II Fuzzy Logic System (IT2FLS) and Spatially Constrained Fish School Optimization algorithm (SCFSO). This technique can readily interfere with and analyze huge datasets and complicated tumors (anomalies), and it may be the proactive estimation for being incorporated or implemented in clinical experience for the benefit of both physicians and the patients, and it may offer doctors with a meaningful experience. The SCFSO-IT2FLS method presented here was implemented on the BRATSSICAS dataset. This approach provided the assessment parameters as Dice overlap index (DOI) with $96 \pm 2.1$ and sensitivity value with $98 \pm 1.1$. These results compared with existing approaches, and this method may be used to segment T1-weighted (T1-W), Flair (Fluid Attenuated Inversion Recovery), and T2weighted (T2-W) MRI sequences of varying axis coordination. This approach allows for the separation of non-tumor (edema) and tumor areas, and such an advantage is the therapeutic pre-planning can be frequently done [1].

Detection of brain tumor and analyses are critical in medical imaging. The work in [2] focused on segmenting anomalies in axial brain MRI DICOM slices since this formation has the benefit of maintaining full metadata. The axial slices were based on the assumption that the right and left halves of the brain were separated by a LoS (Line of Symmetry). A semi-automatic system extracts normal and pathological features from every brain MRI slice in the DICOM analysis. Here, fuzzy clustering (FC) was utilized to extract distinct clusters for varying $\mathrm{k}$ from DICOM slices. After that, the silhouette fitness function was utilized to produce the bestsegmented image with the maximum inter-class permanence.

Morphological procedures improved the tissue class's clustered boundaries even better. The FC approach was coupled with normal image postprocessing approaches like marker regulated watershed (MCW) segmentation, distance regularized level set (DRLS), and region growing (RG). This approach was tested on the wellknown BRATS challenge data set of several methods as well as the clinical data set of axial T2-w MR images. The metadata information contained in the DICOM header was utilized to do quantitative analysis on the slices. These segmentation technique's validation over ground truth images demonstrated that DRLS augmented brain images with FC have the better Dice similarity coefficient and Jaccard score. The average dice and jaccard scores for 10 patient trials in the BRATS dataset for segmenting tumor sections were 0.79 and 0.88 , respectively, and 0.78 and 0.86 for the clinical analysis. Finally, DICOM data was utilized to create $3 \mathrm{D}$ visualizations and estimate tumor volumes [2].

Traditionally, human inspection was used to identify and detect computed tomography and magnetic resonance brain images. Computerized Tomography and Magnetic Resonance Imaging images generate extraneous noise caused by operator output, which can lead to significant classification errors. A new 
technique for Brain Tumor Classification based on the usage of a Back Propagation Neural Networks (BPNN) with Fuzzy C Means (FCM) was presented in [3]. The processes followed were: decision making was divided into two stages like pre-processing and decision-making. Following that, analysis was carried out utilizing high-level segmentation (through FCM) and low-level segmentation (using BPNN). Twentyfive brain tumor images were utilized for assessment [3].

Image segmentation is essential in the domain of medical applications. MRI scans of brain without and with the diseases were recommended for the diagnosis and staging of Alzheimer's disease. Clustering was a popular image segmentation concept that identifies groups so that samples from the same group may be compared to each other rather than samples from different groups. There has recently been a consideration regarding the use of fuzzy clustering techniques, which retain more detail from the input images than clustering theory. The Modified FCM method was used due of its adaptability, since it allows pixels to belong to multiple groups with varied degrees of membership. The MFCM was utilized to establish the clusters, and the Binary Gravitational Search method was employed to improve the segmentation process's efficiency. Various brain topics like Grey Matter (GM), White Matter (WM), the hippocampal regions, and Cerebrospinal Fluids (CSF) were segmented to identify Alzheimer's disease. When compared to other current methods, the BGSA using the MFCM algorithm produced superior results. This method has an accuracy rating of 93.3 percent [5].

Segmentation of tumor using MRI data was a necessary but lengthy manual process carried out by medical specialists. Due to the significant difference in the formation of tumor tissues across individuals, as well as their near similarity to normal tissues, automating this procedure was difficult. MRI was a sort of sophisticated medical imaging that offers precise information on the human's soft tissues. To segment and identify the brain tumor using MRI, several brain tumor segmentation and detection techniques were utilized. The benefits and limitations of these approaches for brain tumor identification and segmentations were analyzed, with an emphasis on illuminating the benefits and limitations of these techniques for brain tumor segmentation and detection. The use of MRI segmentation and detection in various processes was also covered. An overview of several segmentation approaches for identifying brain tumors using MRI images is discussed in [6].

\section{SYSTEM ARCHITECTURE}

From MRI images, brain image segmentation is difficult and complicated, yet it is important for tumor diagnosis and classification, edema, hemorrhage detection, and necrotic tissues. MRI imaging is the most effective imaging technology for detecting abnormalities in brain areas early. MRI image acquisition settings, unlike CT, may be changed to generate high contrast images with varying grey levels for varied instances of neuropathology. As a result, MRI image segmentation is expected to be a hot topic in the medical imaging research community in the near future. MRI is a non-invasive method that may be performed as frequently as needed for brain imaging. MRI scans are used to provide accurate and detailed images of organs from various angles in order to identify any abnormalities. There are two forms of MRI: high field MRI, which produces high-quality images, and low field MRI, which produces the minor diagnostic conditions. MRI images enable doctors to see even hairline fractures and rips in ligament, muscle, and other soft tissue ailments. MRI works on the basis of energy absorptions and emissions in the radio free ranges of the electromagnetic spectrums. MRI was useful for detecting brain disorders like tumor, hemorrhage, multiple sclerosis, or lesion. Accurate anatomical three-dimensional (3D) models developed from 2D MRI medical image data aid in providing accurate and precise diagnosis about spatial relationship among critical anatomical structure like eloquent cortical area, vascular structure, and so on, as well as other pathological findings that would otherwise be unrecognizable by the human eye.

The following section comprise the method meant to identify brain tumors in their early stages and classify them as benign or malignant. Figure 1 depicts the process of system architecture during these periods. 


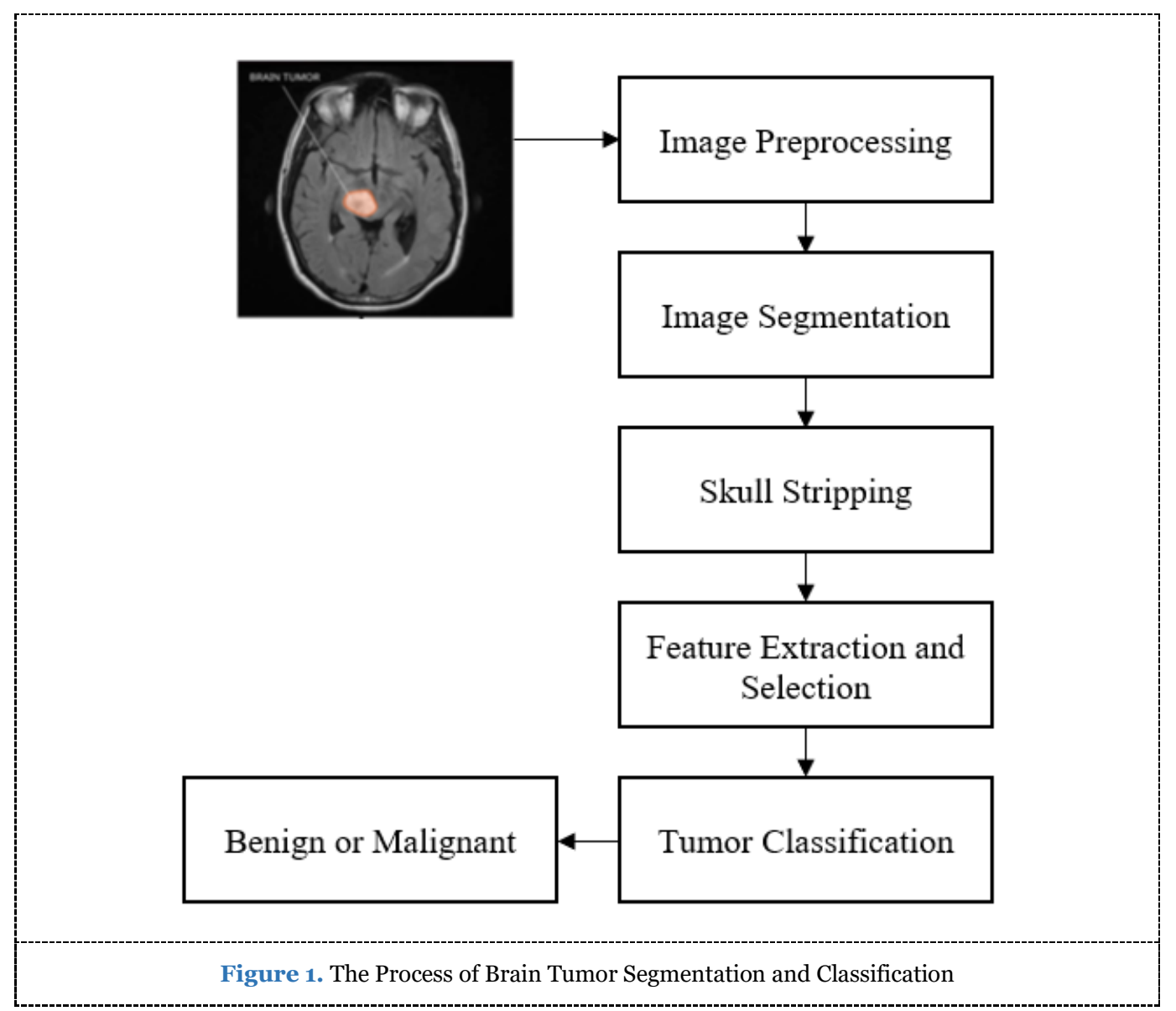

A) Image Processing: It is the process of using a digital computer for processing digital images using the algorithms. As a subset or area of digital signal processing, digital image processing has numerous benefits over analogue image processing. It permits the applications of broader range of algorithm to the input data while avoiding problems like noises and distortions during the process. Due to images are specified in $2 \mathrm{D}$, image processing may be modelled as a multi-dimensional system (or more). Three elements impact the genesis and production of image processing: the first is the computers development; the second is the development of mathematics; and the third is the growth of science (specifically the development and enhancement of discrete mathematical theory). Demand for diverse applications in the environments, agricultures, industry, military, and medical sciences has increased in the third phase.

B) Image pre-processing: Image pre-processing may significantly improve feature extraction and image analysis performance. A frequent stage in many feature descriptor approaches is mathematical normalization of a data collection, which is comparable to image pre-processing.
C) MR Brain Image Skull Stripping: Mathematical morphology-based techniques, atlas-based techniques, deformable surfacebased techniques, intensity-based techniques, and hybrid techniques are the five types of skullstripping methods discussed in the literature. The MRI device generates brain images as $3 \mathrm{D}$ volumetric data represented as a stack of twodimensional slices for diverse brain image applications like study of anatomical structure, volumetric analysis, localization of anatomy, treatment planning, diagnosis, surgical planning, and computer-integrated planning. It is critical to study the information contained in these brain slices using computer-aided techniques.

D) Image Segmentation: Image Segmentation is the splitting of digital images into various subgroups (pixels) process known as Image Objects, which lowers the image's complication and facilitates image analysis. To split and group a specific collection of pixels from an image, several image segmentation techniques were employed. Labels were assigned to pixels as a result, and pixels with the same label are grouped together because they share a characteristic. Borders and draw lines can be used to define and differentiate the most 
essential items in an image from the rest of the less important ones.

E) Feature Extraction: A stage in the dimensionality reduction process that isolates and lowers a large collection of raw data into smaller classifications is feature extraction. As a result, processing would be a lot easier. The most essential element is that these big data sets contain a high number of variables.
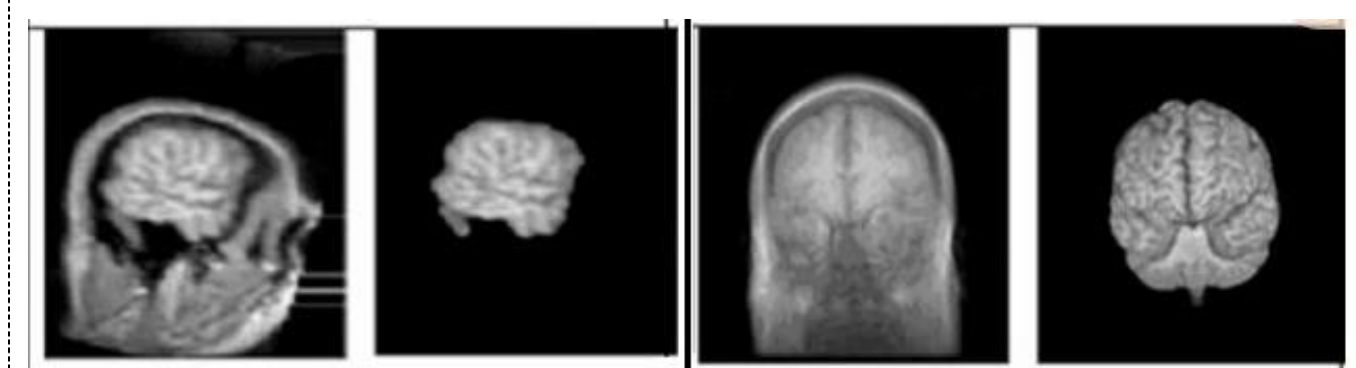
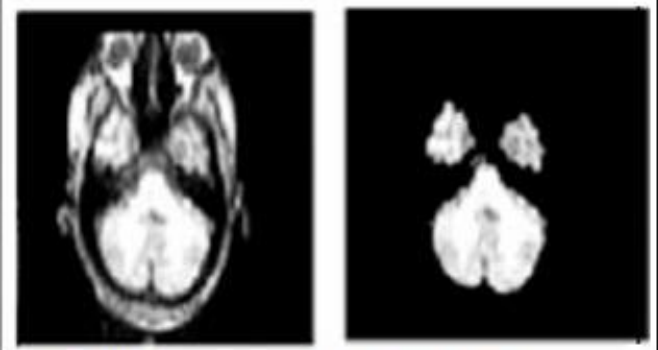

Skull Stripping of 2D Brain Images
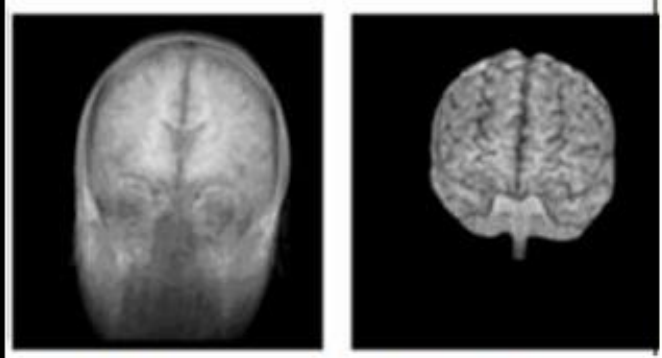

Skull Stripping of 3D Brain Images

Figure 2. The Skull Stripping Process of Brain Images

F) Feature Selection: Feature selection minimizes the amount of input variables while creating the predictive model. The count of input variables must be decreased to minimize modelling computational costs and, in some circumstances, to improve model accuracy.

G) Tumor Classification: Deep learning is one of the most often used approaches in tumor classification to differentiate among benign and malignant types. $\mathrm{CNN}$ is an efficient learning technique for classification issues. Deep neural networks (DNN), particularly convolutional neural networks (CNNs), are frequently employed in image classification applications and have demonstrated considerable performance since 2012. CNN study on medical image classification has produced results comparable to human specialists. CNN outperforms in situations with fewer data and higher dimensionalities. The CNN classifier is capable of distinguishing among benign and malignant.

Recently, image segmentation has been the complex and active study topic in the domain of processing image. Despite the availability of a wide range of cutting-edge approaches for MRI segmentations of brain, segmenting MRI remains the difficult issue, with much room for future researches to increase the precision, accuracy, speed of segmentation techniques. Implementing parallelization and merging diverse algorithms might be the future roadmap for brain segmentation method advancement. Growing new understanding regarding the link between different illnesses and anatomical variation is emerging as a result of continuing study in the biological world. As a result, brain segmentation is becoming more popular as the initial stage in tools for detecting and assessing structural deviance. Alzheimer's disease and Multiple Sclerosis (MS), for example, are conditions that may be investigated based on changes in brain structures.

\section{CONCLUSION AND FUTURE WORK}

This study analyzed an automated system that can correctly identify brain tumors as benign or malignant using MRI scans. Image processing and image segmentation techniques can be applied on MRI images. Both the machine learning and deep learning approaches can be utilized for the classification of brain tumor. If machine learning approaches were used means, during the segmentation phase, brain tumors may be segmented using s-FCM. The SF, FIF, and GLCM approaches may be utilized to extract features, and the PCA methodology can be used to pick features. If the deep learning-based approaches were used means there is no need to use additional techniques for feature extraction and selection. The deep learning techniques were capable of both feature 
extraction and selection. The CNN approach may be utilized in the classification process. The larger the training database, the better the detection efficiency. This study is part of an integrated framework that allows for tumor identification, benign and malignant tumor classification, tumor rating, tumor tracking, and patient care stages. The analysis in future study will be focused on introducing new features and developing an interactive platform.

\author{
ETHICS APPROVAL AND CONSENT TO \\ PARTICIPATE \\ Not applicable.
}

\section{HUMAN AND ANIMAL RIGHTS}

No animals/humans were used for studies that are basis of this research.

\section{CONSENT FOR PUBLICATION}

Not applicable.

AVAILABILITY OF DATA AND MATERIALS

The authors confirm that the data supporting the findings of this research are available within the article.

\section{FUNDING}

None.

\section{CONFLICT OF INTEREST}

The authors declare no conflict of interest, financial or otherwise.

\section{ACKNOWLEDGEMENTS}

The authors would like to thank their present employer for providing support while carrying out this research work.

\section{REFERENCES}

[1]. Alagarsamy S, Yu-Dong Z, Vishnuvarthanan G, Murugan P R, Sakthivel S. Smart Identifications of Topographically Variant Anomalies in Brain Magnetic Resonance Imaging Using a Fish School based Fuzzy Clustering Approach. IEEE Trans Fuzzy Syst. 2020; 12(8): 3165-3177.

[2]. Suresh K, Samiayya D, Vincent PM, Srinivasan K, Jayakody D.N.K, Reina D.G, Inoue A. An Efficient Hybrid Fuzzy-Clustering Driven 3D-Modeling of Magnetic Resonance Imagery for Enhanced Brain Tumor Diagnosis. Electronics. 2020; 9(475): 123.

[3]. Sangeeta S, Ritu K. Brain Tumor Detections Using FCM and BPNN. Int J Basic Appl Biol. 2014; 2(1): 83-88.

[4]. Rajesh Kumar P, Arun Prasath T, Pallikonda Rajasekaran M, Vishnuvarathanan G. Segmentations of Brain Subject in MR Image using Hybrid Segmentation Techniques. Int J Eng Adv Technol. 2019; 9(1S4): 724-728.

[5]. Sudipta R, Sanjay N, Samir K.B, Indra K M. A Review on Automated Brain Tumor Detections and Segmentations from MRI of Brain. Int J Adv Res Comput Sci Softw Eng. 2013; 3(6): 1706-1746.

[6]. Kalavathi P, Prasath V. B. S. Methods on Skulls Stripping of MRI Head Scan Images-A Review. J Digit Imaging. 2016; 23(3): 365-379.
[7]. Hooda H, Verma O.P, Arora S. Optimal Fuzzy C-Means Algorithms for Brain Images Segmentations. Eng Appl Artif Intell. 2019; 1(1): 591-602.

[8]. Valverde S, Oliver A, Roura E, González-Villà S, Pareto D, Vilanova JC, Ramió-Torrentà L, Rovira À, Lladó X. Automated tissues segmentations of MR brain image in the presences of white matter lesion. Med Image Anal. 2017; 35: 446-457.

[9]. Rajesh Kumar P, Arun Prasath T, Pallikonda Rajasekaran M, Vishnuvarathanan G. Brain Subjects Estimations Using PSO and K-Mean Clustering-An Automated Aid for the Assessments of Clinical Dementia. Int Conf Inform Communi Technol Intell Syst. 2017; pp. 482-489.

[10]. Selvy P.T, Palanisamy V, Purusothaman T. Performances Analysis of Clustering Algorithm in Brain Tumor Detections of MR Image. Euro J Sci Res. 2011, 62(3): 321-330.

[11]. Selvanayagi K, Kalugasalaam P. Preprocessing and Enhancements of Brain Magnetic Resonance Images (MRI). Int J Res Comput Appl Management. 2012; 2(10): 47-54. 